\title{
Semiautomatic Detection of Cardiac Diseases employing Dual Tree Complex Wavelet Transform
}

\author{
Navdeep Prashar ${ }^{1}$, Meenakshi Sood ${ }^{2}$, Shruti Jain ${ }^{3}$ \\ ${ }^{1}$ Department of Electronics and Communication Engineering, Bahra University, Solan, 173234, Himachal Pradesh, India \\ 2-3 Department of Electronics and Communication Engineering, Jaypee University of Information Technology, Solan, 173234, \\ Himachal Pradesh, India
}

\begin{tabular}{l}
\hline \hline Article Info \\
\hline Article history: \\
Received Sept 5, 2018 \\
Revised Oct 27, 2018 \\
Accepted Nov 13, 2018
\end{tabular}

\section{Keyword:}

Heart Rate

Morphology Analysis

Discrete Wavelet Transform

Dual-Tree Complex Wavelet

Transform.

\begin{abstract}
Electrocardiogram (ECG) contains lot of information which can be utilized for a mechanism to detect cardiac abnormalities. The ECG signal is too sensitive to various types of noises as it is of low frequency and has weak amplitude, these noises reduce the diagnostic accuracy and may lead to the incorrect decision of the clinician. So, denoising of ECG signal is an essential requirement for an accurate detection of Heart disease. In this paper, a Dual-Tree Complex Wavelet Transform technique (DTCWT) is presented to denoise the noisy ECG signal and to extract the Principal features followed by implementation of Peak Detection Algorithm. The performance is evaluated on the basis of performance metrics and an increase in SNR is achieved using the technique. With the proposed technique, calculated heart rate is in consensus with the gold standard of the various bench mark databases used and accurate heart disease was determined.
\end{abstract}

\author{
Corresponding Author: \\ Navdeep Prashar, \\ Departement of Electronics and Communication Engineering, \\ Bahra University, \\ Distt. Solan H.P, India. \\ Email: nav.prashar@gmail.com
}

\section{Introduction}

ECG indicates an electrical activity of heart which provides cardiologists to extract the useful information about the functioning of the heart [1],[2],[3]. Any disorder in the heart, leads towards the Heart Disease often detected by an ECG signal [4],[5],[6],[7]. Some of the common heart diseases are Coronary, Arrhythmia, and Myocardial Infarction. A correct analysis of ECG features is required for an early detection of Heart disease. But the presence of noise artifacts at different levels can change the signal amplitude and frequency which creates the major problem to detect the actual abnormalities [8],[9],[10].Earlier, feature extraction of an ECG signal analysis has been done by time domain based method, which made researchers difficult to study. Therefore, the signals are studied and processed in frequency domain. In this context, the Fast Fourier Transform (FFT) technique has been introduced which further failed to provide the information regarding an exact location of frequency components. This problem has been resolved by introducing a Short-Term Fourier Transform (STFT) which has limitations of seeking the optimal time-frequency precision. To overcome these limitations, many techniques and algorithms have been developed in recent years for signal denoising. Among all, Discrete Wavelet Transform (DWT) has become an important computational tool for performing signal 
processing. It overcomes the shortcomings of time window size which does not vary with frequency [11],[12],[13],[14]. Further use of DWT for low-frequency biomedical signals noticed some of the limitations such as lack of shift invariance property, aliasing, oscillations and lack of directionality. To overcome these limitations and to provide an efficient tool for signal denoising a Dual-Tree Complex Wavelet Transform (DTCWT) technique has been introduced that has shift invariance property and anti-aliasing effect for biomedical signals [15],[16],[17]. Some of the methods such as Fourier transform infrared spectroscopy (FTIR), Nuclear magnetic resonance spectroscopy (NMR), Fluorescence spectroscopy are used for Nanoparticle and Protein interaction in biomedical applications [18].

This paper outlines the DTCWT technique to denoise the ECG signal and Peak Detection Algorithm for an accurate detection of peaks along with computation of peak intervals. The DTCWT technique has been evaluated based on performance metrics such as the Signal to Noise Ratio (SNR), Mean Square Error (MSE), and Percent Root Mean Square Difference (PRD). By comparing the performance parameters of the presented technique with other techniques, DTCWT was found well suited for an ECG denoising. Later different databases are used to verify the diseases on the basis of Heart rate (bpm) and RR interval (sec). Table 1 signifies the Heart diseases related to Peak morphology which indicates various diseases at particular Heart Beat rate, complexes, and intervals. This paper is further organized as: section 2 explains methodology which is followed by the experimental results and their comparison with existing papers in Section 3 and concluded in section 4.

Table 1. Symptoms of different Heart Diseases based on Complexes and intervals

\begin{tabular}{|c|c|}
\hline Diseases related with QRS wave & Symptoms on ECG wave \\
\hline Bradycardia & $H R<60 \mathrm{bpm}, P R$ interval: $0.12-0.20 \mathrm{sec}, R R$ interval:0.6-1.2 \\
\hline Atrial Tachycardia & $100 \mathrm{bpm}<H R<175 \mathrm{bpm}$, Missing $P$ wave, $R R$ interval $<0.6 \mathrm{sec}$ \\
\hline Atrial flutter & $250 \mathrm{bpm}<H R<400 \mathrm{bpm}, Q R S$ : less than $0.10 \mathrm{sec}$ \\
\hline Supra Ventricular Tachycardia & $\begin{array}{c}150 \mathrm{bpm}>H R>250 \mathrm{bpm}, \text { Prolonged } Q T \text { interval, } P \text { wave difficult to } \\
\text { visualize, } R R \text { interval }<0.6 \mathrm{sec}\end{array}$ \\
\hline Sick Sinus Syndrome & $H R<40 \mathrm{bpm}$ \\
\hline Premature Ventricular Contraction & Skipped Heartbeat, $Q R S>0.12 \mathrm{sec}$ \\
\hline Sinus Tachycardia & $100 \mathrm{bpm}<H R<180 \mathrm{bpm}, Q R S<0.10, P R$ interval $: 0.12-0.20 \mathrm{sec}$ \\
\hline Sinus Arrhythmia & $60 \mathrm{bpm}<H R<100 \mathrm{bpm}, Q R S<0.10, P R$ interval $: 0.12-0.20 \mathrm{sec}$ \\
\hline Ventricular flutter & $H R>250 \mathrm{bpm}$ \\
\hline Ventricular Tachycardia & $H R>100 \mathrm{bpm}, R R$ interval $<0.6 \mathrm{sec}$ \\
\hline Normal Rhythm & $60 \mathrm{bpm}<H R<100 \mathrm{bpm}, 0.6 \mathrm{sec}<R R$ interval $<1.2 \mathrm{sec}$ \\
\hline
\end{tabular}

\section{Methodology}

Input signal of ECG category i.e. MIT-BIH Arrhythmia v5 is taken from Physio Bank ATM database of 100 records [19]. This input ECG signal has length of 1536 samples which is sampled at rate of $360 \mathrm{~Hz}$. The whole process of implementation of DTCWT technique along with peak detection algorithm with the help of flow graph is shown in "Fig.1".

2.1 Preprocessing Stage- Input ECG signal was fed to digital FIR High pass filter using Blackman windowing technique to remove Base Line Wander noise with cut-off frequency $0.5 \mathrm{~Hz}$. The order of filter is taken $N=10$ by defining the window length i.e. $N+1=11$ [20]. 
2.2 Denoising Stage - ECG signal is a non-stationary signal that cannot be denoised using normal filter. In most recent studies, wavelet transform has been used to denoise these non-stationary signals by virtue of its high performance and qualitative characteristics. In this work, DTCWT has been employed for this stage as explained in the following section.

2.2.1 Signal decomposition using Dual Tree Complex Wavelet Transform (DTCWT)-DTCWT has been applied at the output obtained from preprocessing stage. This technique is composed of two DWTs in which first DWT give real part and second DWT gives the imaginary part. These two real DWTs use two different sets of filter with each satisfies Perfect Reconstruction (PR) conditions. The two set of real filters are jointly designed so that wavelet is approximately analytic resulted coefficients are complex numbers which is expressed by "equation (1)".

$$
\Psi(t)=\Psi_{h}(t)+j \Psi_{g}(t)
$$

where $\Psi_{\mathrm{h}}(t)$ values are real and even part while $\Psi_{\mathrm{g}}(t)$ values are real and odd such that

$$
\Psi_{g}(t) \approx H \Psi_{h}(t)
$$

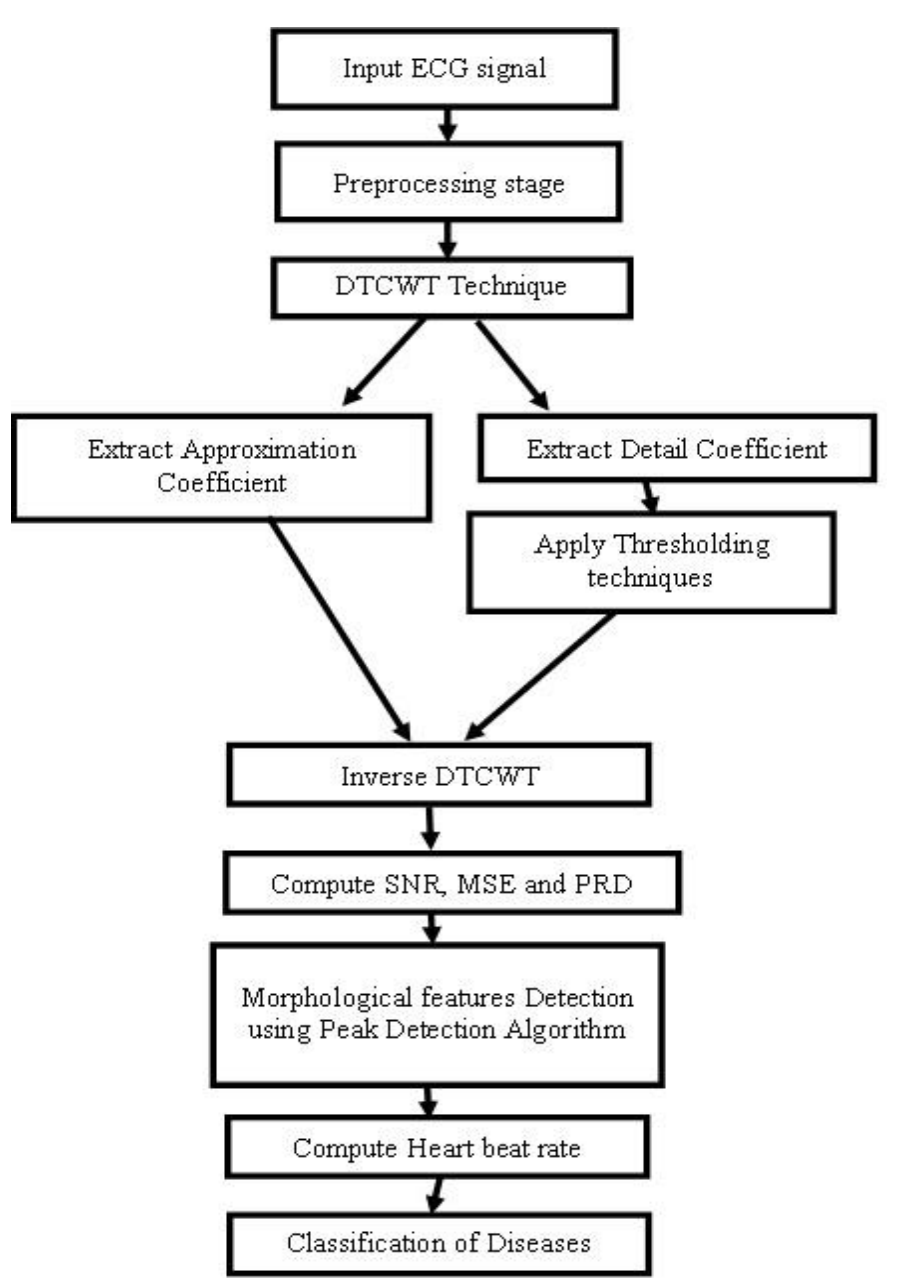

Figure1. Flow Graph 
where $H$ is the Hilbert transform. In DTCWT, output is computed by two filter banks; each filter bank consists of two high pass and low pass filter pair as shown in "Fig.2". Two times transformation leads to expansion of the DWT coefficients, because $N$ coefficients in DWT turn into $2 N$ coefficients in DTCWT. In "Fig. 2", $x$ is the input signal, $h_{\mathrm{o}}(\mathrm{n})$ is the low pass filter output and $h_{1}(\mathrm{n})$ constitutes High pass filter for upper filter bank and $g_{0}(\mathrm{n})$ and $g_{1}(\mathrm{n})$ denotes Low pass and High pass filter pair respectively for lower filter bank. Each stage comprises of two digital filters and two down samplers by 2 to produce digital signal. The down sampled output of $h_{0}(\mathrm{n})$ and $h_{1}(\mathrm{n})$ give approximation coefficient and detail coefficient for upper filter bank respectively. Approximation coefficient are low frequency components called as scaling function which were extracted at the end of set level of decomposition whereas, detail coefficient are high frequency components known as wavelet functions. Similarly, output of low pass filter $g_{0}(n)$ and high pass filter $g_{1}(\mathrm{n})$ give approximation coefficient and detail coefficient for lower filter bank respectively. The approximation coefficient is further decomposed and the process continues till the set level of decomposition. In this process, DTCWT technique is implemented up to 4 level of decomposition to compute the absolute value of the real and imaginary coefficients. Complex detail coefficients have been extracted at each level of decomposition scale which provides 4 detail sub bands designated as weight 1 complex (wt1Com, wt 2 Com, $w t 3 \mathrm{Com}$ and wt $4 \mathrm{Com}$ ). Similarly, complex approximation coefficients are extracted as wt5Com sub band. The details of coefficients at each sub band of approximation level and detail level are depicted in Table 2. The number of decomposition level is chosen such that classification of signal is retained in the wavelet coefficient.

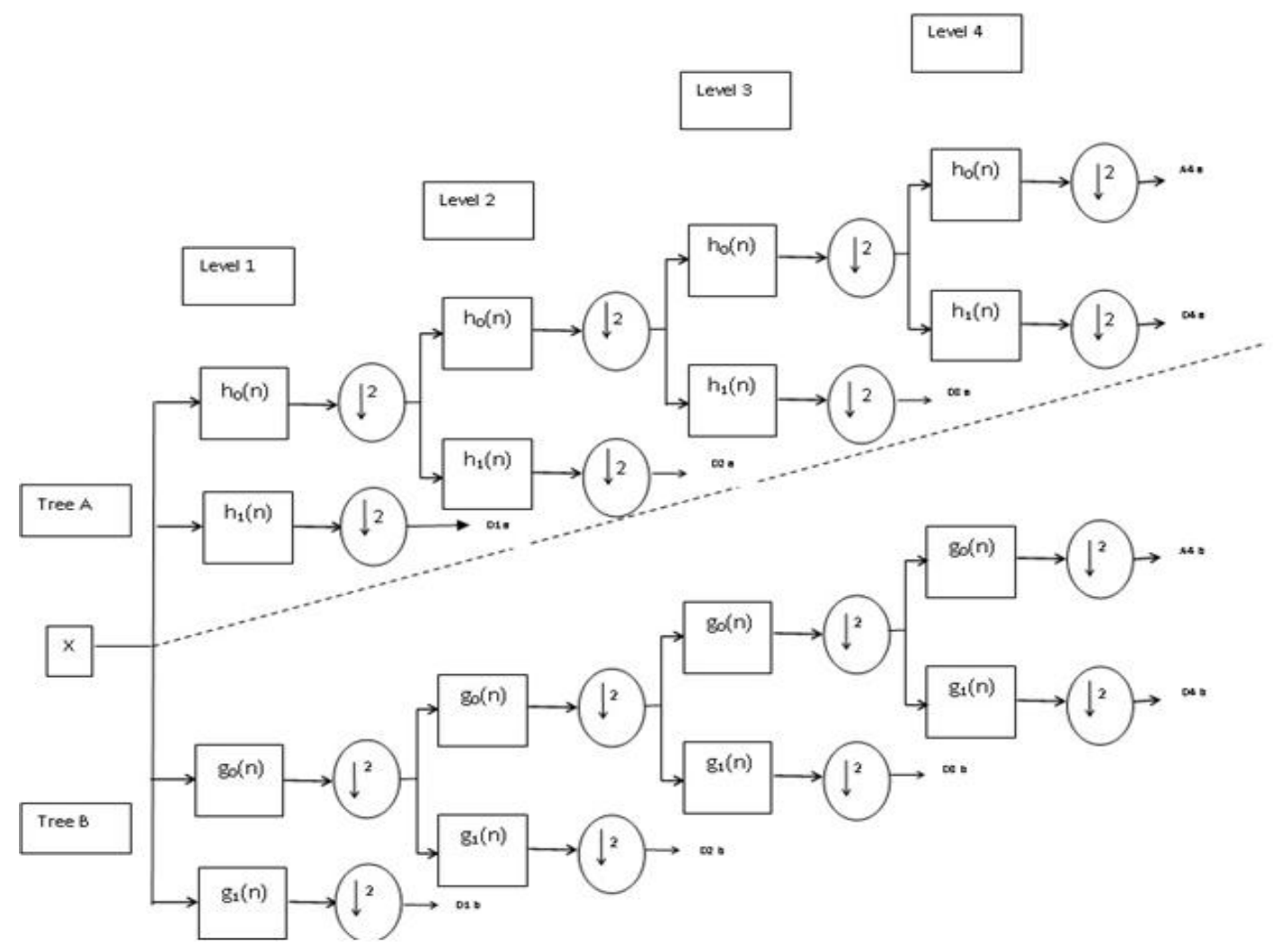

Figure 2. Multilevel decomposition using DTCWT 
Table 2. Coefficient at each sub band of Detail and Approximation level assuming sampling frequency $\left(f_{\mathrm{s}}\right) 360 \mathrm{~Hz}$

\begin{tabular}{|c|c|c|c|}
\hline S.No. & $\begin{array}{c}\text { Sampling } \\
\text { Frequency at each } \\
\text { decomposition level }\end{array}$ & $\begin{array}{c}\text { Sub Bands of Detail and Approximation } \\
\text { Coefficients (upper filter bank + lower } \\
\text { filter bank) }\end{array}$ & $\begin{array}{c}\text { Number of } \\
\text { Coefficients } \\
\text { (In Complex form) }\end{array}$ \\
\hline 1. & 180 & $w t 1$ Com $=d_{1 \mathrm{a}}+i d_{1 \mathrm{~b}}$ & 768 \\
\hline 2. & 90 & $w t 2$ Com $=d_{2 \mathrm{a}}+i d_{2 \mathrm{~b}}$ & 384 \\
\hline 3. & 45 & $w t 3$ Com $=d_{3 \mathrm{a}}+i d_{3 \mathrm{~b}}$ & 96 \\
\hline 4. & 22.5 & $w t 4$ Com $=d_{4 \mathrm{a}}+i d_{4 \mathrm{~b}}$ & 96 \\
\hline 5. & 22.5 & $w t 5$ Com $=a_{4 \mathrm{a}}+i a_{4 \mathrm{~b}}$ & \\
\hline
\end{tabular}

2.2.2 Thresholding Technique-Thresholding technique has been applied to every detail coefficient to denoise the data [21]. Many thresholding techniques have been developed in literature which includes Donoho Method [22], Neigh Block, Savitzky method by [23], Hard thresholding and Soft thresholding. A robust estimation method of noise level $\delta$ is proposed by Donoho and Jhonstone based on Median Absolute Deviation (MAD) has been applied in this paper. Noise level $\delta$ is calculated by the "equation (3)".

$$
\delta=\left(\operatorname{median}\left\{\left|\chi_{i v}\right|\right\} / 0.6745\right)
$$

where $\chi_{\text {iv }}$ represent the detail coefficients at finest level. Threshold value $\lambda$ is calculated using "equation (4)" which is expressed as

$$
\lambda=\delta \sqrt{2 \log (k)}
$$

where $k$ is the size of signal at particular detail level. After computing the threshold value for each decomposition level, soft thresholding technique is applied at each level to remove the noise given by "equation (5)".

$$
Y=\operatorname{sign}(x) \cdot(|x|-\lambda)
$$

where $Y$ is the output, and $x$ is the input value. In soft thresholding, the coefficients in magnitude greater than threshold value are shrunk towards zero by subtracting the threshold value from coefficient value. 
2.2.3 Inverse DTCWT-The inverse DTCWT is computed on the threshold wavelet coefficients to obtain the denoised signal.

2.2.4 Performance Parameter Evaluation- The performance metrics such as Signal to Noise Ratio (SNR), Mean Square Error (MSE) and Percent Root Mean Square Difference (PRD) are computed to analyze the denoising process. The best results are achieved when the recovered signal has highest SNR, smallest PRD and has lowest MSE.

2.3 Morphological Feature Extraction using Peak Detection Algorithm-Morphological features of an ECG signal such as amplitude and locations of $P, Q, R, S, T$ peaks and their intervals has been detected using Peak Detection Algorithm.

\section{Peak Detection Algorithm}

Input - Pre-processed signal $(Y)$

Output - Peak Detection

1. Define Sample Rate

2. Detection of $R$ peak : The point with maximum amplitude gives the $R$ peak location.

If $R$ peak is located for each cycle $\left(R_{\text {peak }}\right)$, then different other peaks are detected by the following methods:

3. Detection of $P$ peak $: P$ peak location detection is given as

$$
\begin{gathered}
a=\left[R_{\text {peak }}-\left(\frac{\text { Sample Rate } \times 25}{\text { Beat }}\right)\right]:\left[R_{\text {peak }}-\left(\frac{\text { Sample } \text { Rate } \times 5}{\text { Beat }}\right)\right] \\
P \text { peak amplitude }=\max (Y(a))
\end{gathered}
$$

4. Detection of $Q$ peak $: Q$ peak location detection is given as

$$
\begin{gathered}
b=\left[R_{\text {peak }}-\left(\frac{\text { Sample } \text { Rate } \times 10}{\text { Beat }}\right)\right]:\left[R_{\text {peak }}-\left(\frac{\text { Sample Rate } \times 1}{\text { Beat }}\right)\right] \\
Q \text { peak amplitude }=\min (Y(b))
\end{gathered}
$$

5. Detection of $S$ peak $: S$ peak location detection is expressed as

$$
\begin{gathered}
c=\left[R_{\text {peak }}+\left(\frac{\text { Sample Rate } \times 1}{\text { Beat }}\right)\right]:\left[R_{\text {peak }}+\left(\frac{\text { Sample Rate } \times 5}{\text { Beat }}\right)\right] \\
S \text { peak amplitude }=\min (Y(c)) ;
\end{gathered}
$$

6. Detection of $T$ peak: $T$ peak location detection is represented as 


$$
\begin{gathered}
d=\left[R_{\text {peak }}+\left(\frac{\text { Sample Rate } \times 10}{\text { Beat }}\right)\right]:\left[R_{\text {peak }}+\left(\frac{\text { Sample Rate } \times 30}{\text { Beat }}\right)\right] \\
T \text { peak amplitude }=\max (Y(d))
\end{gathered}
$$

End

Computation of Heart Beat Rate- Finally, Heart beat rate of denoised signal is computed using "equation (6)".

$$
\text { Heart Rate }=\frac{\text { Sample Rate } \times 60}{R_{2}-R_{1}}
$$

where $R_{2}$ and $R_{1}$ represents the location of two subsequent $\mathrm{R}$ peaks on time scale

\section{Results and discussions}

In this section methodology which is explained in Section 2 was implemented using MATLAB. First stage is preprocessing stage. In preprocessing stage, output of an ECG signal has been obtained after removing baseline wander noise as shown in "Fig.3". In second stage, detailed and approximate coefficients are calculated by applying DTCWT technique on preprocessed signal as shown in "Fig.4" and "Fig.5". After applying the proposed algorithm for removing the noise from ECG signal, inverse DTCWT is applied to reconstruct the signal shown in "Fig.6". The performance parameters SNR, MSE and PRD of denoised signal using proposed algorithm are tabulated in Table 3.

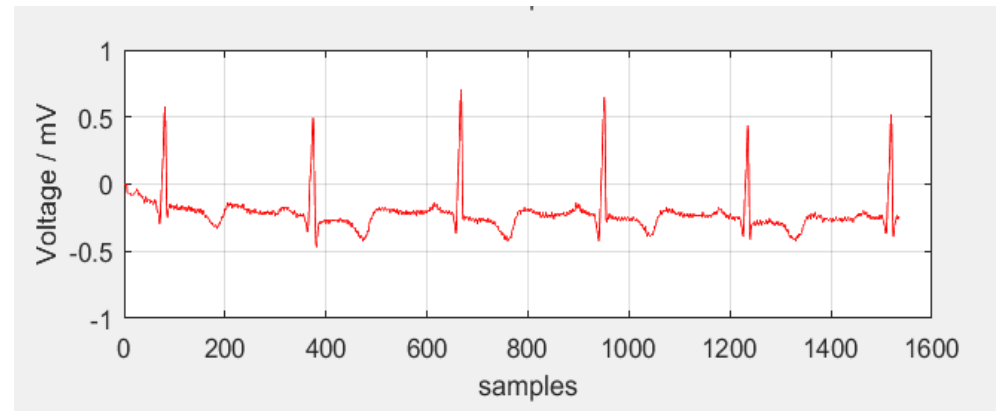

Figure3. Time domain analysis of ECG signal filtered by FIR High Pass filter using Blackman window

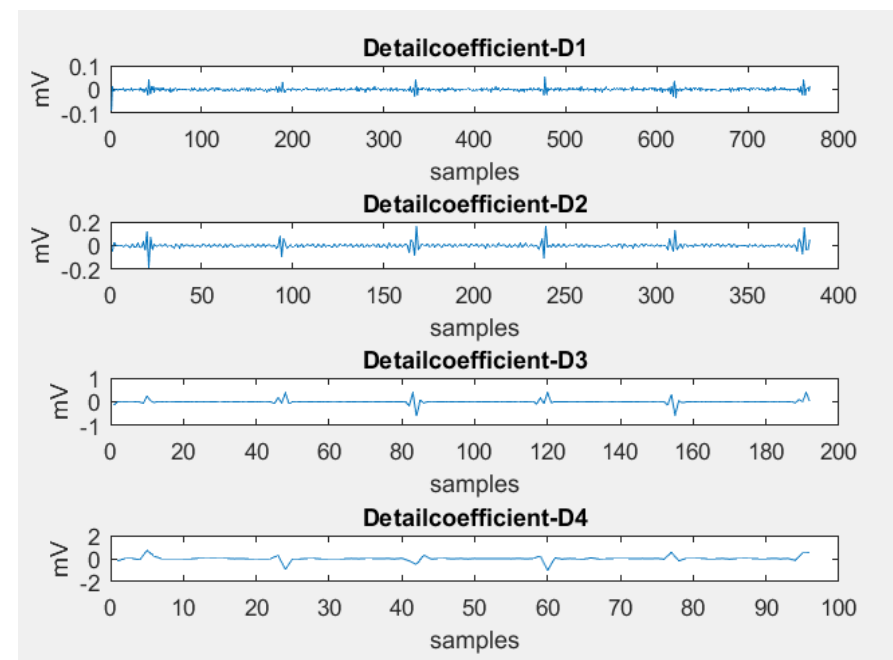

Figure 4. Detailed coefficients using DTCWT at different levels. 


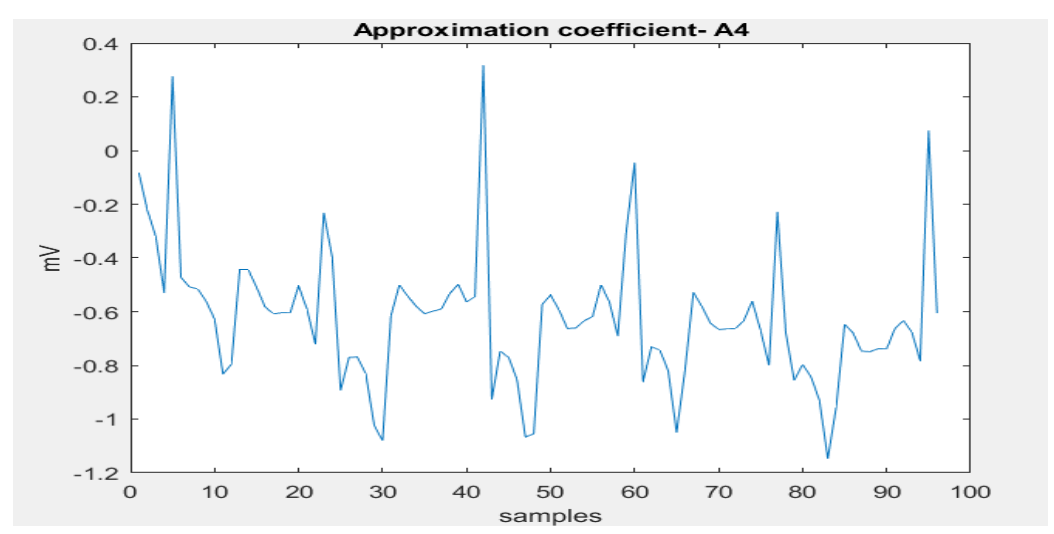

Figure 5. Approximation coefficient using DTCWT at level 4

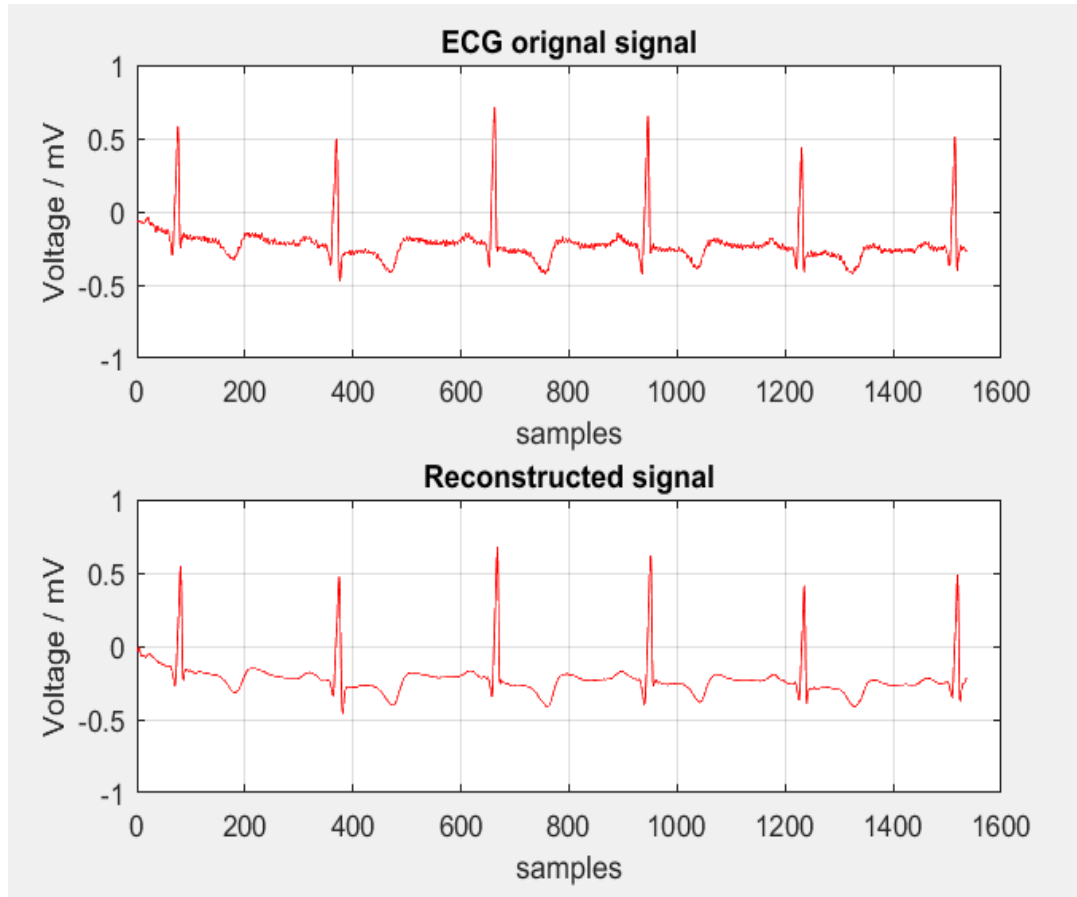

Figure 6. ECG original signal and Reconstructed signal

Table 3. Performance Parameters of Denoised Signal Using DTCWT

\begin{tabular}{|c|c|c|c|c|c|}
\hline S.No. & $\begin{array}{c}\text { Wavelet } \\
\text { Family }\end{array}$ & $\begin{array}{c}\text { SNR of original } \\
\text { signal }(\mathrm{dB})\end{array}$ & $\begin{array}{c}\text { SNR of } \\
\text { denoised } \\
\text { signal }(\mathrm{dB})\end{array}$ & MSE & PRD \\
\hline 1. & DTCWT & -4.9666 & 27.7500 & $1.0777 \mathrm{e}-04$ & 0.0410 \\
\hline
\end{tabular}




\subsection{Comparison table with previous work}

The results obtained by our proposed algorithm are compared with the previous research papers are shown in Table 4. On comparing the performance metrics of proposed technique with other techniques, the comparative result shows that proposed technique is well suited for denoising any ECG signal.Later, different peaks of the denoised and reconstructed ECG signal have been detected using Peak Detection Algorithm as shown in "Fig.7".

Table 4. Comparison with Existing Techniques

\begin{tabular}{|c|c|c|c|c|}
\hline S.No. & Technique & SNR (dB) & MSE & PRD \\
\hline 1. & Proposed Technique & 27.7500 & $1.0777 \mathrm{e}-04$ & 0.0410 \\
\hline 2. & DWT [23] & 26.5396 & $1.4172 \mathrm{e}-04$ & 0.0471 \\
\hline 3. & Neigh Block [17] & 4.2177 & 0.62052 & 4.8693 \\
\hline 4. & Savitzky [17] & 2.0434 & 1.28079 & 6.9956 \\
\hline
\end{tabular}

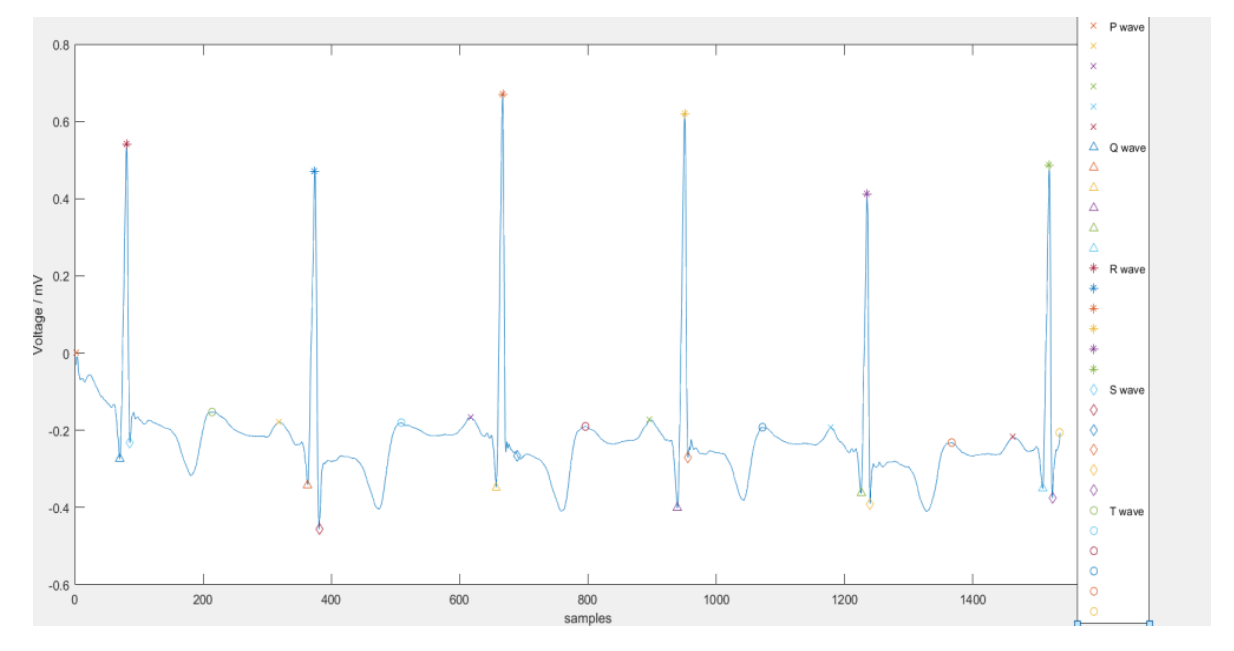

Figure 7. Detection of peaks of Denoised signal using Peak detection algorithm

Different peak intervals like $R-R$ interval, $P-Q$ interval, $Q R S$ complex and $S$ - $T$ interval at each cycle of denoised ECG signal has been computed and, average of every interval is taken which is depicted in Table 5. Table also gives the comparative result of intervals and complexes using two techniques (DWT and DTCWT). 
Table 5. Calculation of Different Peak Intervals

\begin{tabular}{|c|c|c|c|}
\hline S.No. & Types of Intervals & $\begin{array}{c}\text { DWT Technique } \\
(\mathrm{sec})\end{array}$ & $\begin{array}{c}\text { DTCWT Technique } \\
(\mathrm{sec})\end{array}$ \\
\hline 1. & $R-R$ interval & 0.8021 & 0.7989 \\
\hline 2. & $P-Q$ interval & 0.1333 & 0.1329 \\
\hline 3. & $P-R$ interval & 0.1628 & 0.1620 \\
\hline 3. & $Q R S$ complex & 0.0517 & 0.0523 \\
\hline 4. & $S-T$ interval & 0.3479 & 0.2852 \\
\hline
\end{tabular}

Heart beat rate is computed using DTCWT technique and on comparing this value with the given range of MIT-BIH Arrhythmia database, we observe that the resultant value falls in the range which is illustrated in Table 6.

Table 6. Heart Beat Rate Computation

\begin{tabular}{|c|c|c|}
\hline \multirow{2}{*}{$\begin{array}{c}\text { Heart Beat Rate per } \\
\text { minute }\end{array}$} & $\begin{array}{c}\text { Heart Beat Rate computation } \\
\text { using DTCWT technique. }\end{array}$ & $\begin{array}{c}\text { Heart Beat Rate mentioned in } \\
\text { MIT-BIH Arrhythmia } \\
\text { Database (Gold Standard) [18] }\end{array}$ \\
\cline { 2 - 3 } & 75.1219 & $70-89$ \\
\hline
\end{tabular}

We have already classified the different diseases based on HR and RR interval expressed in Table 1. On the basis of obtained results of Heart rate and RR interval of different standard databases, nature of type of disease related to heart have been computed and tabulated in Table 7.

Table 7. Types of diseases related to HR and RR interval for different ECG databases

\begin{tabular}{|c|c|c|c|}
\hline Database & $\begin{array}{c}\text { Heart Rate } \\
(\mathrm{bpm})\end{array}$ & $\begin{array}{c}\text { RR interval } \\
(\mathrm{sec})\end{array}$ & Disease \\
\hline $\begin{array}{c}\text { MIT/BIH } \\
\text { Arrhythmia(MLII) } 100 \\
\text { record }\end{array}$ & 75.1219 & 0.7989 & Normal Rhythm \\
\hline $\begin{array}{c}\text { MIT/BIH Arrhythmia } \\
\text { (MLII) } 103 \text { record }\end{array}$ & 161.0799 & 0.4278 & Atrial Tachycardia \\
\hline $\begin{array}{c}\text { MIT/BIH Arrhythmia } \\
\text { (MLII) } 106 \text { record }\end{array}$ & 204.8729 & 0.4452 & $\begin{array}{c}\text { Supra Ventricular } \\
\text { Tachycardia }\end{array}$ \\
\hline $\begin{array}{c}\text { MIT/BIH Noise Stress Test } \\
\text { (MLII) }\end{array}$ & 58.7456 & 1.10 & Bradycardia \\
\hline
\end{tabular}

In next phase, different optimization algorithms like Cuckoo-search, particle swarm optimization, differential evolution and artificial bee colony algorithms [24],Swarm inspired evolutionary algorithm ,Numerical function optimization [25] and Elephant herding optimization (EHO) [26] will be implemented on different ECG databases to find a subset of ECG features from larger feature pool to achieve better classification performance.

\section{Conclusion}

This paper presents the denoising of an ECG signal using Dual Tree Complex Wavelet Transform technique. Simulation results with different techniques, shows that our proposed algorithm has the highest SNR, smallest PRD and lowest MSE. This paper also outlines the extraction of different features like fiducial point localization, time intervals measurements, and morphology analysis using Peak Detection Algorithm on the 
proposed denoised and reconstructed signal. Later different databases are used for calculation of heart rate and RR interval for determining the accurate heart disease.

\section{References}

[1] J. G. Webster (Ed.), "Medical Instrumentation, Application and Design". John Wiley \& Sons, 2001.

[2] R. Gupta, S. Singh, K. Garg, S. Jain, "Indigenous Design of Electronic Circuit for Electrocardiograph", International Journal of Innovative Research in Science, Engineering and Technology, 3(5), 1213812145, May 2014.

[3] N. Prashar, S. Jain, M. Sood, J. Dogra, "Review of biomedical system for high performance applications",4th IEEE International Conference on signal processing and control (ISPCC 2017), Jaypee University of Information technology, Waknaghat, Solan, H.P, India, pp 300-304, September 21-23, 2017.

[4] B. Pandey and R. B. Mishra, "An integrated intelligent computing method for the detection and interpretation of ECG based cardiac diseases", International Journal of Knowledge Engineering and SoftData Paradigms", vol. 2, pp. 182-203, 2010.

[5] A. Dhiman, A. Singh, S. Dubey, S. Jain, "Design of Lead II ECG Waveform and Classification Performance for Morphological features using Different Classifiers on Lead II". Research Journal of Pharmaceutical, Biological and Chemical Sciences (RJPBCS),7(4), 1226- 1231: July-Aug 2016.

[6] C. C. Chiu, T. H. Lin, and B. Y. Liau, "Using correlation coefficient in ECG waceform for arrhythmia detection", Biomedical Engineering Applications, Basis and Communications, vol. 17, pp. 147-152, 2005.

[7] A. Saini, N. Kumar, A. Raj, S. Jain, "Performance Analysis of Cascaded Denoising Block for ECG Signal Analysis using Different Filters", Proceedings of the 12th INDIACom;5th International Conference on Computing for Sustainable Global Development, Bharati Vidyapeeth's Institute of Computer Applications and Management (BVICAM), New Delhi (INDIA) March 14th - 16th, pp 22512256, 2018.

[8] G. M. Friesen, T. C. Jannett, M. A. Jadallah, S. L. Yates, S. R. Quint, and H. T. Nagle, "A Comparison of the Noise Sensitivity of Nine QRS Detection Algorithms" IEEE Transactions on Biomedical Engineering, vol. 37, pp. 85-98, 1990.

[9] V. X. Afonso, W. J. Tompkins, T. Q. Nguyen, and S. Luo, "ECG Beat Detection Using Filter Banks", IEEE Transactions on Biomedical Engineering, vol. 46, pp. 192-201,1999.

[10] A. B. M. Aowlad Hossain and M. A. Haque, "Analysis of noise sensitivity of different ecg detection algorithms", International Journalof Electrical and Computer Engineering, vol. 3, no. 3, pp. 307-16, 2013.

[11] V.Sukanya, C. Saritha, Y. Narasimha Murthy, "ECG Signal Analysis Using Wavelet Transforms", Bulg.J.Phys.68-77,2008.

[12] S. Jain, "Classification of Protein Kinase B Using Discrete Wavelet Transform",.International Journal of Information Technology, 10(2), 211-216, 2018.

[13] T. Cai, B. Silverman, "Incorporating information on neighbouring coefficients into wavelet estimation", Sankhya, Vol 63, no. Series B,2001.

[14] H. N Niranjana Murthy, M. Meekashi, "ECG Signal Denoising and Ischemic Event Feature Extraction using Daubechies Wavelets",International Journal of Computer application Vol.67,No.2,April 2013.

[15] I. W. Selesnick, R. G.Baraniuk, and N. G. Kingsbury, "The dual-tree complex wavelet transform, ” IEEE Signal Processing Magazine, vol. 22,no. 6, pp.123-151, 2005.

[16] F. Wang and Z. Ji, "Application of the Dual-tree Complex Wavelet Transform in Biomedical Signal" Bio-Medical Materials and Engineering, vol. 24, pp. 109-115, 2014.

[17] F.M. Ghombavani, K. Kiani , "A powerful novel method for ECG signal de-noising using different thresholding and Dual Tree Complex Wavelet Transform",2nd International Conference on Knowledge Based Engineering and Innovation (KBEI), pp.966-971, November, 2015.

[18] Jasmin Šutkovi, Amina Jašarević, "A review on Nanoparticle and Protein interaction in biomedical applications", Periodicals of engineering and natural sciences, Vol. 4 No. $2,2016$.

[19] https://www.physionet.org/physiobank/database/html/mitdbdir/records.htm.

[20] N. Prashar, J. Dogra, M. Sood, S. Jain, "Removal of electromyography noise from ECG for high performance biomedical systems", Network Biology. 8(1),12-24, 2018. 
[21] J. Dogra, M. Sood, S. Jain, N. Prashar, "Segmentation of magnetic resonance images of brain using thresholding techniques", $4^{\text {th }}$ IEEE International Conference on signal processing and control (ISPCC 2017), Jaypee University of Information technology, Waknaghat, Solan, H.P, India, pp. 311-315, September 21-23, 2017.

[22] G. Georgieva-Tsaneva and K. Tcheshmedjiev, "Denoising of Electrocardiogram Data with Methods of Wavelet Transform,"International Conference on Computer Systems and Technologies, pp. 9-16, 2013.

[23] A. Awal, S. S. Mostafa, and M. Ahmad, "Performance Analysis of Savitzky-Golay Smoothing Filter Using ECG Signal," IJCIT, vol. 1, no. 2, pp. 24-29, 2011.

[24] Civicioglu, E. Besdok, "A conceptual comparison of the Cuckoo-search, particle swarm optimization, differential evolution and artificial bee colony algorithms," Artif. Intell. Rev. ,1-32,2013.

[25] Turker TUNCER,"SCSO: A novel sine-cosine based swarm optimization algorithm for numerical function optimization,"Periodicals of engineering and natural sciences, Vol. 6 No. $2,2018$.

[26] Aboul Ella Hassanien, Moataz Kilany and Essan H. Houssein ,"Combining Support Vector Machine and Elephant Herding Optimization for Cardiac Arrhythmias,"arXiv:1806.08242v1[eee.SP],June 20,2018.

\section{BIBLIOGRAPHY OF AUTHORS}

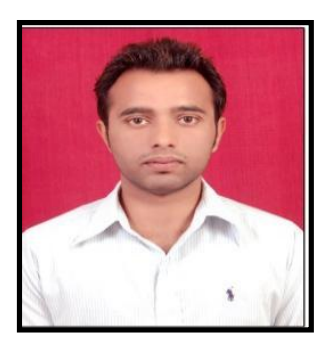

Navdeep Prashar has obtained his Bachelor of Technology degree from CTIEMT, Jalandhar and Master of Technology degree from Centre for Development of Advanced Computing (CDAC), Mohali, in 2010 and 2012 respectively. He is currently serving as an Assistant Professor in Bahra University Shimal Hills and also pursuing Ph.D from JUIT Solan. He has 6+ years of teaching experience to both undergraduate and postgraduate students. Prashar has published one book and many papers in the International Journal and Conferences. His current interest includes Biomedical applications, Low Power techniques, VLSI Design \& Testing, and System on Chip.

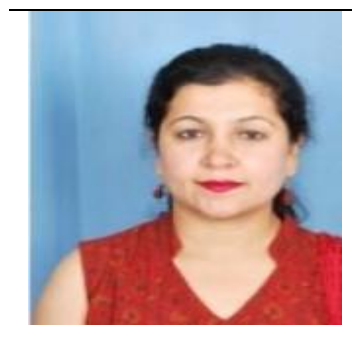

Dr. Meenakshi Sood is Senior Assistant Professor in the Department of Electronics and Communication Engineering at Jaypee University of Information Technology, Waknaghat, H.P, India and received her Ph. D in Biomedical Signal Processing. She is Gold Medalist and has been awarded Academic Award for her performance in Master of Engineering (Hons.) from Panjab Univeristy, Chandigarh. Her research areas interests are Image and Signal processing, Antenna Design, Metamaterials and Soft Computing. She has published more than 25 papers in reputed journals and 30 papers in International conferences. She is a senior member of IEEE.

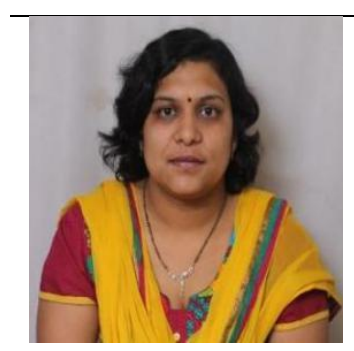

Dr. Shruti Jain is Associate Professor in the Department of Electronics and Communication Engineering at Jaypee University of Information Technology, Waknaghat, H.P. She has a teaching experience of around 13 years and before joining JUIT, she worked as Assistant Professor in Haryana Engineering College, Jagadhari, Ambala College of Engineering, Ambala. She has specialization in Biomedical Signal Processing, Computer- Aided design of FPGA and VLSI circuits, combinatorial optimization. She has published more than 50 papers in reputed journals and 30 papers in International conferences. She is a senior member of IEEE, life member of Biomedical Engineering Society of India and member of IAENG 